\title{
ChemComm
}

Check for updates

Cite this: Chem. Commun., 2018, 54,180

Received 24th August 2017,

Accepted 26th October 2017

DOI: $10.1039 / \mathrm{c7cc06651a}$

rsc.li/chemcomm

\section{Mechanochemical synthesis of cooperative spin crossover materials $\dagger$}

\author{
Jed H. Askew (D) and Helena J. Shepherd (D) *
}

\begin{abstract}
We describe the synthesis of switchable spin crossover materials via mechanochemistry for the first time. Three chemically diverse spin crossover materials have been produced using solvent-free grinding. Crucially, cooperative spin transition behavior and crystallinity is retained, presenting exciting opportunities for the discovery of new materials with switchable magnetic, optical and structural properties.
\end{abstract}

Spin crossover (SCO) materials have seen a surge in interest in recent years as a result of their optical, magnetic and structural bistability in technologically accessible temperature ranges, giving rise to a number of potential applications in sensing, display and actuator technologies. ${ }^{1,2}$ The bistability in these systems stems from their propensity to switch reversibly between high-spin (HS) and low-spin (LS) states in response to physical stimuli including temperature, pressure, light irradiation, guest molecules and magnetic field. ${ }^{3}$ There are hundreds of known SCO-active materials in the literature including molecular coordination complexes, 1D coordination polymers and 2-/3-dimensional metal organic frameworks. The cooperativity of the SCO phenomenon (abruptness of the transition, hysteretic effects etc.) is a result of electron-phonon coupling between SCO centers in the solid state through elastic interactions in the lattice. ${ }^{4}$ The degree of cooperativity is important for application, and has been shown to be sensitive to the crystallinity of the material, particle size and the presence of solvents in the lattice. ${ }^{2,5}$ Synthesis of bulk SCO materials has typically been carried out via traditional solution state chemistry, although more complex techniques for the production of nanoparticles and thin films have been developed in the last decade. ${ }^{6}$ Remarkably, all existing techniques use solvents and can be timeconsuming; often requiring inert atmospheres and multiple synthetic steps. This aspect is particularly limiting in the search for new SCO-active materials. Herein, we present for the first time the rapid

School of Physical Sciences, University of Kent, Canterbury, CT2 7NH, UK.

E-mail:h.j.shepherd@kent.ac.uk

$\dagger$ Electronic supplementary information (ESI) available: Experimental details, variable temperature Raman spectroscopy, FTIR spectra and discussion of the effect of washing the mechanochemically prepared samples. See DOI: 10.1039/ c7cc06651a and facile synthesis of three chemically different classes of SCO material, using mechanochemical techniques. We show that microcrystalline samples of SCO-active molecular materials, 1D coordination networks and 3D MOFs can be produced using this technique and crucially the cooperative behaviour of the spin transition is maintained. Finally, we highlight how this technique can be used to accelerate SCO research.

Mechanochemistry refers to the reaction of materials through the application of mechanical energy, often through grinding in the solid state. While mechanochemistry has long been used in the synthesis of inorganic materials and composites, in recent years it has also been applied in the synthesis of molecular systems, coordination complexes and frameworks, co-crystals and supramolecular networks. ${ }^{7,8}$ Mechanistic explanations for the success of the mechanochemical approach are numerous and as yet no single model can be applied to the varied range of systems that can be produced in this manner. However, it is clear that the technique has the potential to produce both known and new materials across diverse areas of chemistry.

Triazole-based 1D SCO coordination polymers have become the subject of a huge number of studies owing to their high temperature range of operation and relative ease of chemical modification, making them extremely attractive from the point of view of application. ${ }^{9}\left[\mathrm{Fe}(\mathrm{atrz})_{3}\right] \mathrm{SO}_{4}$ (where atrz $=4$-amino- 1,2 4-triazole, compound $\mathbf{1}$ ) is a member of this family and has been previously prepared using solution-state techniques. ${ }^{10}$ Like other members of this family it shows a pronounced colour change from purple in the LS state at ambient temperature, to white in the HS state above $350 \mathrm{~K}$. For the first time, we have used mechanochemical techniques to prepare samples of 1; $\left[\mathrm{NH}_{4}\right]_{2}\left[\mathrm{Fe}\left(\mathrm{SO}_{4}\right)_{2}\right] \cdot 6 \mathrm{H}_{2} \mathrm{O}$ and atrz were ground in a pestle and mortar without solvent for 5 minutes.

Within 30 seconds, traces of the purple colour indicative of the LS product began to appear. After $c a .4$ minutes the entire sample was purple and no further colour change was observed at room temperature. The product became damp as a result of liberation of water molecules from the hydrated metal salt. It dried out during a further minute of grinding, and was subsequently 


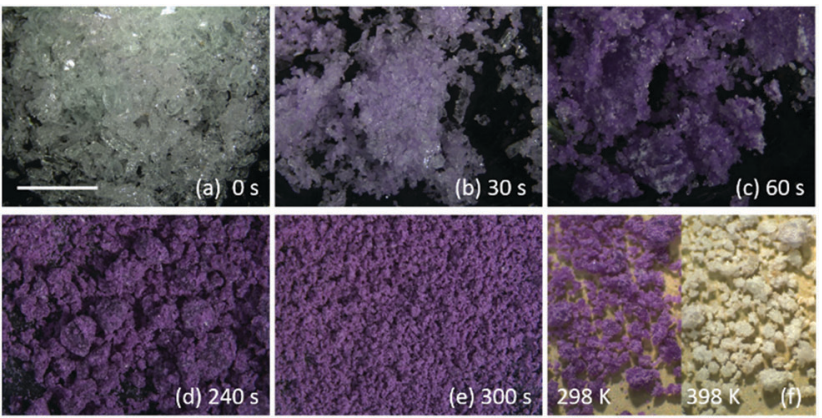

Fig. 1 Pictures of the mechanochemical reaction to form 1 at room temperature as a function of duration of grinding. (a) Shows the reagents mixed in the pestle and mortar prior to grinding. (b-e) Show the change in colour accompanying the formation of the LS product. Scale bar represents $5 \mathrm{~mm}$ for panels (a)-(e). (f) Shows the colour change associated with the LS $\rightarrow$ HS transition on warming from $298 \mathrm{~K}$ (left) to $398 \mathrm{~K}$ (right).

further dried by heating. $\S$ This general procedure was followed for the production of a series of SCO-active materials, experimental details and materials characterization are provided in the ESI. $\dagger$ Fig. 1 shows the reaction progression as a function of grinding time, and the characteristic purple (LS) $\rightarrow$ white (HS) colour change accompanying SCO on heating is clear in Fig. 1(f).

Despite the increasingly routine nature of mechanochemical synthesis in modern chemistry, there are no reports of these techniques being used to make switchable SCO materials. The most likely reason for this is the widely-held assumption that grinding results in significant reduction in cooperativity of the spin transition. There are many examples in the literature of powdered and nano-scale SCO materials exhibiting weaker cooperativity than associated bulk samples. This is attributed to amorphisation and a reduction in the coherent domain length of individual particles, through increased defects and a more significant surface contribution. ${ }^{11}$ Cooperativity can be assessed through evaluation of the abruptness of the SCO curve and hysteresis widths via magnetometry. ${ }^{12}$ Previous studies on the effects of grinding SCO materials involved grinding solutionsynthesized molecular SCO materials for several hours. This resulted in a pronounced reduction in cooperative behaviour and the eventual suppression of all SCO properties. ${ }^{13,14}$ Thus, careful comparison of SCO properties arising from mechanochemical and solution-state synthetic protocols (via SQUID magnetometry) is required to ensure functionality of the produced materials is not deleteriously affected by grinding.

The SCO behaviour of solution-synthesized $\mathbf{1}$ (denoted $\mathbf{1}_{\text {sol }}$ ) as a function of temperature is shown in Fig. 2(a); the spin transition is abrupt and accompanied by a hysteresis of $23 \mathrm{~K}$ for the first cycle $\left(T_{\frac{1}{2}} \uparrow=350 \mathrm{~K}, T_{\frac{1}{2}} \downarrow=327\right)$, and $14 \mathrm{~K}$ on subsequent cycles. By comparison, the mechanochemically synthesized sample after annealing (denoted $\mathbf{1}_{\text {mech }}$ ) has very similar hysteresis widths of $21 \mathrm{~K}$ for the first cycle $\left(T_{\frac{1}{2}} \uparrow=348 \mathrm{~K}, T_{\frac{1}{2}} \downarrow=327 \mathrm{~K}\right)$, and $14 \mathrm{~K}$ on subsequent cycles (Fig. 2(b)). Clearly, despite grinding the sample for several minutes during cooperative SCO behaviour; the spin transitions in the two samples are virtually indistinguishable. The degree of crystallinity is comparable in both $\mathbf{1}_{\text {mech }}$ and $\mathbf{1}_{\text {sol }}$, as demonstrated in the powder diffraction patterns in Fig. 2(d).
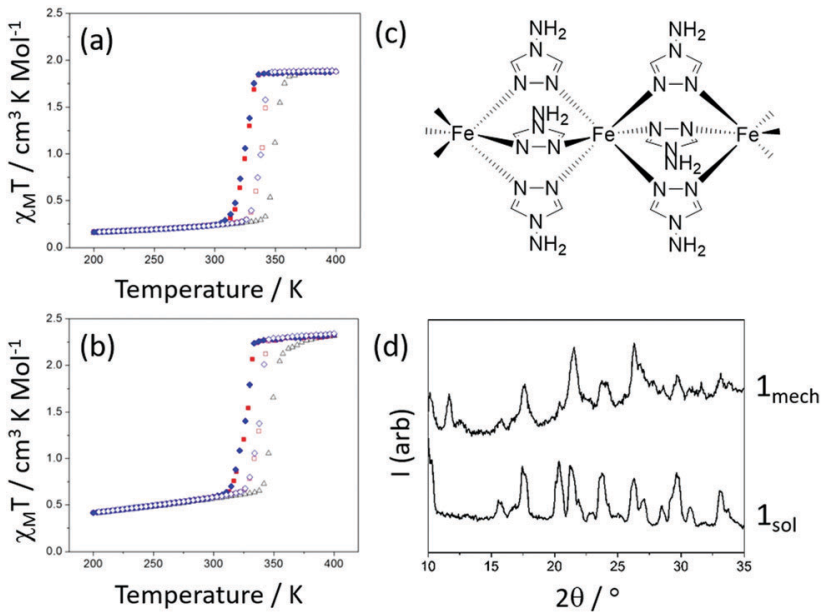

Fig. 2 (a) $\chi_{M} T$ versues $T$ for $\mathbf{1}_{\text {sol }}$ (3 cycles), (b) $\mathbf{1}_{\text {mech }}$, cooling and heating are shown as closed and open symbols respectively, (c) structure of the cationic 1D chain observed in $\mathbf{1}$. (d) XRD patterns of $\mathbf{1}$ sol and $\mathbf{1}_{\text {mech }}$.

There are however additional peaks in the powder diffraction pattern, which we attribute to irreversible structural differences, possibly caused by water loss from the lattice. This is discussed further in the ESI. $\dagger$ Raman spectroscopy (shown in the ESI $\dagger$ ) of the sample, in both HS and LS states, reveals typical changes in the spectrum associated with SCO. ${ }^{15}$ A sample of the related $\left[\mathrm{Fe}(\text { atrz })_{3}\right]\left(\mathrm{BF}_{4}\right)_{2}$ complex was also synthesized using mechanochemistry and shows similar cooperativity to the equivalent solution-state product. More details are provided in the ESI. $\dagger$

One of the most frequently studied $\mathrm{Fe}^{2+} \mathrm{SCO}$ molecular materials is $\left[\mathrm{Fe}(\text { phen })_{2}(\mathrm{NCS})_{2}\right]$ (where phen = 1,10-phenanthroline, compound 2). At room temperature it is in the HS state and undergoes SCO to the LS state around $178 \mathrm{~K}$, as shown in Fig. 3(a). ${ }^{16}$ The abruptness of the transition has been shown to be sensitive to varying crystallinity that results from differing preparation methods. It is known that the SCO-inactive complex $\left[\mathrm{Fe}(\text { phen })_{3}\right](\mathrm{NCS})_{2}\left(\mathbf{2}_{\text {pre }}\right)$ can be converted to compound 2 via thermolysis. ${ }^{17,18}$ Mechanochemistry was used to synthesize $\mathbf{2}_{\text {pre }}$ and it was subsequently thermally converted to the active compound $\mathbf{2}_{\text {mech }}$. The SCO properties of both the precursor $\mathbf{2}_{\text {pre }}$ and $\mathbf{2}_{\text {mech }}$ are shown in Fig. $3(\mathrm{~b})$. While $\boldsymbol{2}_{\text {pre }}$ is not SCO-active, $\mathbf{2}_{\text {mech }}$ shows very similar SCO behaviour to the literature values $\left(T_{\frac{1}{2}} \uparrow=T_{\frac{1}{2}} \downarrow=174 \mathrm{~K}\right)$. Fig. $3(\mathrm{~d})$ shows the evolution from $\boldsymbol{2}_{\text {pre }}$ to $\boldsymbol{2}_{\text {mech }}$ with powder diffraction; additional peaks in the $\mathbf{2}_{\text {pre }}$ diffraction pattern are attributed to the presence of by-products from the mechanochemical reaction. Thermal gravimetric analysis of the conversion from $\boldsymbol{2}_{\text {pre }}$ to $\boldsymbol{2}_{\text {mech }}$ is shown in the ESI. $\dagger$ Again, it is clear that $\mathbf{2}_{\text {mech }}$ is crystalline and that cooperative SCO behaviour is retained.

A series of bimetallic ( $\mathrm{Fe}-\mathrm{M}^{\mathrm{I}}$ and $\mathrm{Fe}-\mathrm{M}^{\mathrm{II}}$ ) cyanide-bridged 2- and 3-D coordination polymers known as the Hofmann-like networks represent another class of SCO materials that have been studied extensively. Their sensitivity to guest molecules, huge potential for chemical modification and often high-temperature, cooperative SCO behaviour has attracted increasing attention. ${ }^{19}$ $\left[\mathrm{Fe}(\mathrm{pz})\left\{\mathrm{Au}(\mathrm{CN})_{2}\right\}_{2}\right]$ (where pz = pyrazine, compound 3) is an example of one of these Hofmann-like networks, adopting an infinite 3D structure through ditopic pyrizine ligands, which bridge between 


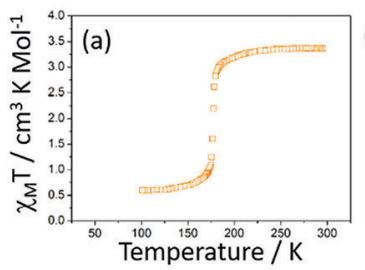

(c)
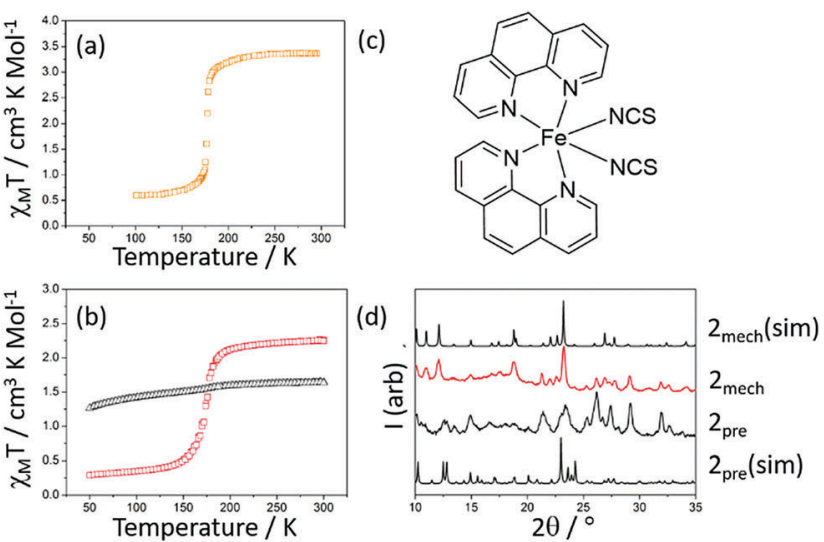

Fig. 3 (a) $\chi_{M} T$ versus $T$ for $\boldsymbol{2}_{\text {sol }}$ adapted from ref. 16 (b) $\chi_{M} T$ versus $T$ for $\boldsymbol{2}_{\text {pre }}$ (black) and $\mathbf{2}_{\text {mech }}$ (red), (c) structure of $\mathbf{2}$ and (d) powder diffraction patterns of [Fe(phen) $\left.)_{3}\right](\mathrm{NCS})_{2}$ (simulated from single crystal data, $\left.\boldsymbol{2}_{\text {pre }}(\mathrm{sim})\right) \mathbf{2}_{\text {pre, }} \mathbf{2}_{\text {mech }}$ and $\left[\mathrm{Fe}(\text { phen })_{2}(\mathrm{NCS})_{2}\right]$ (simulated from single crystal data, $\boldsymbol{2}_{\text {mech }}(\operatorname{sim})$ ).

iron tetracyanoaurate layers. When produced through solutionstate synthetic procedures $\left(\mathbf{3}_{\text {sol }}\right)$ it has an abrupt $\mathrm{SCO}$ above room temperature, as shown in Fig. 4 (a) $\left(T_{\frac{1}{2}} \uparrow=369, T_{\frac{1}{2}} \downarrow=349 \mathrm{~K}\right) .{ }^{20}$ The mechanochemically synthesized complex (denoted $\mathbf{3}_{\text {mech }}$ ) shows very similar SCO behaviour $\left(T_{\frac{1}{2}} \uparrow=370, T_{\frac{1}{2}} \downarrow=354 \mathrm{~K}\right)$.

It must be added at this stage, that due to the nature of the mechanochemical procedure, the final product may well contain unreacted starting materials and by-products from the reaction. While washing the product may remove these impurities, SCO materials are notoriously sensitive to the presence of solvents. For this reason, in this study we aim to demonstrate that SCO-active materials can be produced without any solvent, either in synthesis or processing of the material. As a consequence the accuracy of the absolute values of $\chi_{\mathrm{M}} T$ presented here for systems elaborated through mechanochemistry may be variable depending on the specific impurities present. Of more importance to the current study are the abruptness and hysteresis effects as they relate to cooperativity in the solid state. Further discussion of the effects of washing mechanochemically prepared samples and
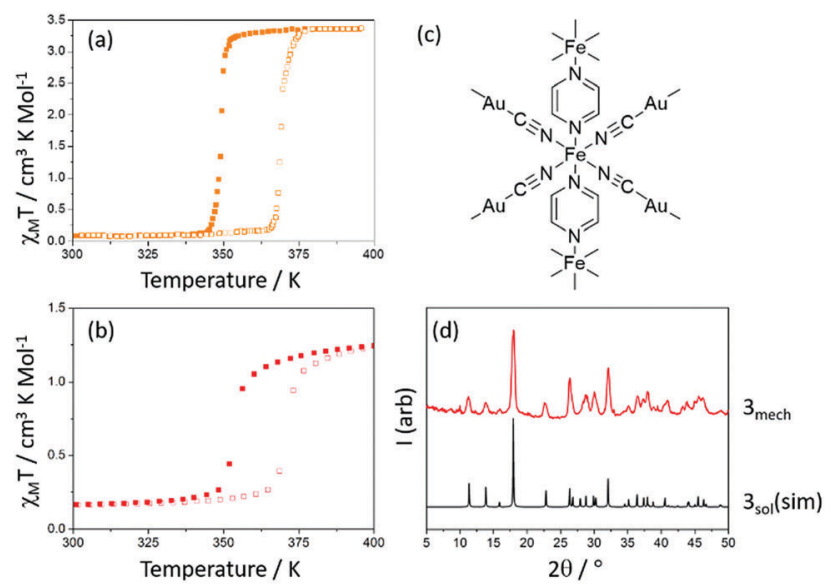

Fig. 4 (a) $\chi_{M} T$ versus $T$ for $3_{\text {sol }}$ adapted from ref. 18 (b) $\chi_{M} T$ versus $T$ for $\mathbf{3}_{\text {mech }}$ (c) structure of $\mathbf{3}$ and (d) PXRD of complex $\mathbf{3}$ (simulated from single crystal data $\left.{ }^{20}\right)$, and $\mathbf{3}_{\text {mech. }}$
Table 1 Transition temperatures, hysteresis widths and "smoothness" values for complexes A-D prepared via mechanochemical and traditional solvent-based routes

\begin{tabular}{lllll}
\hline Sample & $T_{\frac{1}{2}} \downarrow(\mathrm{K})$ & $T_{\frac{1}{2} \uparrow(\mathrm{K})}$ & $\Delta T_{\frac{1}{2}}(\mathrm{~K})$ & Smoothness $^{a} / \mathrm{K}$ \\
\hline 1 $_{\text {sol }}$ & 325 & 338 & 13 & 12 \\
1 $_{\text {mech }}$ & 325 & 338 & 13 & 9 \\
2 $_{\text {sol }}$ & 178 & 178 & 0 & 4 \\
2 $_{\text {mech }}$ & 174 & 174 & 0 & 16 \\
3 $_{\text {sol }}$ & 349 & 369 & 20 & 1 \\
3 $_{\text {mech }}$ & 354 & 370 & 16 & 10
\end{tabular}

${ }^{a}$ Defined in ref. 21 as the difference in the temperatures for which $80 \%$ and $20 \%$ of the molecular complexes are in the HS state. ${ }^{b}$ Values calculated based on data presented in ref. 16. ${ }^{c}$ Values calculated based on data presented in ref. 20.

experimental examples are presented in the ESI. $\dagger$ Values for the transition temperatures of each of the mechanochemically synthesized samples and the equivalent solution-state material are summarised in Table 1. Transition temperatures for all compounds are very similar for each sample, and more importantly, hysteresis widths $\left(\Delta T_{\frac{1}{2}}\right)$ of the samples made via mechanochemistry are identical (in the cases of $\mathbf{1}$ and 2) or very similar (in the case of 3) to those synthesized in solution. While the width of the hysteresis is often used as a proxy for cooperativity in SCO systems, it is also important to look at how abrupt the transition is. Hence Table 1 also shows values for the "smoothness" of the transition (defined as the difference in the temperatures for which $80 \%$ and $20 \%$ of the molecular complexes are in the HS state), a measure which has previously been used to quantitatively compare how abrupt a given transition is. ${ }^{21}$ In the case of $\mathbf{1}_{\text {mech }}$, the transition is slightly more abrupt than that synthesized by solution-state methods (smoothness values of 9 and $12 \mathrm{~K}$ respectively). By contrast, the reverse is true for $\mathbf{2}$ and $\mathbf{3}$, with smoothness increasing by 12 and $9 \mathrm{~K}$ respectively between solution and mechanochemical synthesis protocols. We attribute these differences to reduction in the coherent domain length of particles in the mechanochemically synthesized products. The dependence of hysteresis and smoothness on particle size has been shown to vary for different families of SCO complexes. In triazole-based coordination polymers (such as $\mathbf{1}$ ), hysteresis width and abruptness of the transition is maintained below $10 \mathrm{~nm}^{22,23}$ While in complex 2, the transition becomes much more gradual as size of particles decreases in the nanometre range. ${ }^{18,24} \mathrm{~A}$ similar reduction in abruptness and hysteresis width has also been observed for the family of bimetallic frameworks to which 3 belongs. ${ }^{25,26}$ All observations shown in Table 1 agree with these studies, and point to the reduction in hysteresis and increased smoothness being a consequence of particle size effects rather than something intrinsic to the mechanochemical procedure. Full characterisation of particle size and morphology of $\mathbf{1}$ and $\mathbf{2}$ is currently underway, and we believe this method may present a promising new route towards the size-selective synthesis of SCO materials.

In addition to cooperativity and size-control, some current challenges in the field of SCO include the discovery of active new materials, enhancement of switching and sensing properties and issues associated with technological application. The mechanochemical procedure is extremely rapid compared to solution-state 
techniques, and thus shows great potential for discovery of novel materials through the ability to screen many more combinations of ligand and metal salt. It is also trivial to introduce stoichiometric proportions of potential guest molecules (either liquid or solid) into the mechanochemical procedure to further assess the influence of host-guest interactions on SCO properties. There are no limitations associated with solubility/compatibility of solvent mixtures, and mechanochemistry has even been used to synthesize materials and polymorphs that are not accessible at all via solution. ${ }^{27}$ All of these advantages open the door to development of new materials with improved SCO properties for technological application. As SCO materials move closer towards such real world application, the ability to scale-up syntheses and reduce reliance on solvents (green chemistry) will become ever more important. In this regard mechanochemistry can offer significant opportunities as there are already several commercial routes to scaling up including ball-milling and extrusion. $^{28}$ Having shown that it is possible to synthesize SCO materials while retaining cooperative properties via mechanochemistry we anticipate that many of the advantages brought by the technique will be exploited in the quest for new, technologically useful smart materials.

\section{Conflicts of interest}

There are no conflicts to declare.

\section{Notes and references}

\$CAUTION: Do not use mechanochemical techniques for the preparation of potentially explosive materials. Perchlorate salts and tetrazole ligands have the potential to explode when handled dry, particularly on contact or grinding, and should thus not be used in routine mechanochemical experiments. Care should also be taken when preparing cyanometallate complexes to avoid accidental release of cyanide. $\S$ Compounds $\mathbf{1}_{\text {mech }}, \mathbf{2}_{\text {pre }}$ and $\mathbf{3}_{\text {mech }}$ were prepared by neat grinding of powdered precursors in the absence of solvent. In general, the reagents were manually ground using an agate or glass pestle and mortar for between two and five minutes. $\mathbf{1}_{\text {mech }}$ was heated at $473 \mathrm{~K}$ for 10 hours to remove residual water. $\mathbf{2}_{\text {pre }}$ was converted to $\mathbf{2}_{\text {mech }}$ via heating at $473 \mathrm{~K}$ for 10 hours. $\mathbf{3}_{\text {mech }}$ was used as prepared. Full details are provided in the ESI. $\dagger$

1 K. Senthil Kumar and M. Ruben, Coord. Chem. Rev., 2017, 346, 176-205. 2 M. D. Manrique-Juárez, S. Rat, L. Salmon, G. Molnár, C. M. Quintero, L. Nicu, H. J. Shepherd and A. Bousseksou, Coord. Chem. Rev., 2016, 308, 395-408.
3 M. A. Halcrow, Spin-Crossover Materials: Properties and Applications, Wiley-Blackwell, 1st edn, 2013.

4 P. Gütlich, Y. Garcia and H. A. Goodwin, Chem. Soc. Rev., 2000, 29, 419-427.

5 A. B. Gaspar and B. Weber, in Molecular Magnetic Materials: Concepts and Applications, ed. B. Sielucka and D. Pinkowisz, Wiley VCH, 2016, pp. 231-252.

6 H. J. Shepherd, G. Molnár, W. Nicolazzi, L. Salmon and A. Bousseksou, Eur. J. Inorg. Chem., 2013, 653-661.

7 S. L. James, C. J. Adams, C. Bolm, D. Braga, P. Collier, T. Friščić, F. Grepioni, K. D. M. Harris, G. Hyett, W. Jones, A. Krebs, J. Mack, L. Maini, A. G. Orpen, I. P. Parkin, W. C. Shearouse, J. W. Steed and D. C. Waddell, Chem. Soc. Rev., 2012, 41, 413-447.

8 T. Friščić, Chem. Soc. Rev., 2012, 41, 3493.

9 O. Roubeau, Chem. - Eur. J., 2012, 18, 15230-15244.

10 L. G. Lavrenova, O. G. Shakirova, V. N. Ikorskii, V. A. Varnek, L. A. Sheludyakova and S. V. Larionov, Russ. J. Coord. Chem., 2003, 29, 22-27.

11 J. Larionova, L. Salmon, Y. Guari, A. Tokarev, K. Molvinger, G. Molnár and A. Bousseksou, Angew. Chemie - Int. Ed., 2008, 47, 8236-8240.

12 M. A. Halcrow, Chem. Soc. Rev., 2011, 40, 4119.

13 E. W. Müller, H. Spiering and P. Gütlich, Chem. Phys. Lett., 1982, 93, $567-571$.

14 M. S. Haddad, W. D. Federer, M. W. Lynch and D. N. Hendrickson, J. Am. Chem. Soc., 1980, 102, 1468-1470.

15 J. A. Wolny, R. Diller and V. Schünemann, Eur. J. Inorg. Chem., 2012, 2635-2648.

16 B. Gallois, J. A. A. Real, C. Hauw and J. Zarembowitch, Inorg. Chem., 1990, 29, 1152-1158.

17 E. C. Ellingsworth, B. Turner and G. Szulczewski, $R S C A d v ., 2013,3$, 3745-3754.

18 J. Laisney, A. Tissot, G. Molnár, L. Rechignat, E. Rivière, F. Brisset, A. Bousseksou and M. L. Boillot, Dalton Trans., 2015, 44, 17302-17311.

19 M. C. Muñoz and J. A. Real, Coord. Chem. Rev., 2011, 255, 2068-2093.

20 I. A. Gural'skiy, B. O. Golub, S. I. Shylin, V. Ksenofontov, H. J. Shepherd, P. R. Raithby, W. Tremel and I. O. Fritsky, Eur. J. Inorg. Chem., 2016, 3191-3195.

21 P. Guionneau, M. Marchivie, G. Bravic, J.-F. Létard and D. Chasseau, Spin Crossover in Transition Metal Compounds II. Topics in Current Chemistry, Springer Berlin Heidelberg, Berlin, Heidelberg, 2004, vol. 234, pp. 97-128.

22 J. R. Galán-Mascarós, E. Coronado, A. Forment-Aliaga, M. MonrabalCapilla, E. Pinilla-Cienfuegos and M. Ceolin, Inorg. Chem., 2010, 49, 5706-5714.

23 M. Giménez-Marqués, M. Luisa García-Sanz de Larrea and E. Coronado, J. Mater. Chem. C, 2015, 7946, 7946-7953.

24 F. Javier Valverde-Muñoz, A. B. Gaspar, S. I. Shylin, V. Ksenofontov and J. Real, Inorg. Chem., 2015, 54, 7906.

25 V. Martínez, I. Boldog, A. B. Gaspar, V. Ksenofontov, A. Bhattacharjee, P. Gütlich and J. A. Real, Chem. Mater., 2010, 22, 4271-4281.

26 F. Volatron, L. Catala, E. Rivière, A. Gloter, O. Stéphan and T. Mallah, Inorg. Chem., 2008, 47, 6584-6586.

27 J.-L. Do and T. Friščić, ACS Cent. Sci., 2016, 3, 13-19.

28 D. E. Crawford and J. Casaban, Adv. Mater., 2016, 28, 5747-5754. 